\title{
Development and Utilization of Reversed Phase High Performance Liquid Chromatography Methods for a Series of Therapeutic Agents
}

\section{Jason Olbrich* and Joel Corbett}

Poly-Med, Inc., Anderson, SC, USA

\begin{abstract}
Capability to quantify drug release is vital in many areas of pharmaceutical research. Accurate, rapid and repeatable detection of pharmaceutical agents allows for comparisons between drug delivery constructs prior to in vivo studies as well as identification of therapeutic levels in samples both in vitro and in vivo. Perhaps the foremost scientific method for monitoring of therapeutics is High Performance Liquid Chromatography. This process allows for clear and simple quantification of therapeutic presence in a sample, with an inherent or induced chromophore, through the detection of bound specimen to the utilized column. Multiple drugs can be used through this process and exemplary methods are presented for the pharmaceutics; cefuroxime, clindamycin, dexamethasone, dicloxacillin, doxycycline, metronidazole, oxymetazoline, paclitaxel, tobramycin, and vancomycin. Each of these drugs is analyzed using Reversed-Phase High Performance Liquid Chromatography utilizing a hydrophobic column. Independent, repeatable methods are developed for each drug. Where necessary a pre-column derivatization is used to allow for visualization of otherwise undetectable tobramycin. In the case of each drug, a standard curve is presented to show the linearity of response for the proposed method within a range of absorbance. This work shows the ability to analyze multiple drugs with a single simple, quick, cost effective system and detection methods are evaluated for each drug with a $\mathrm{R}^{2}$ value of greater than 0.99 .
\end{abstract}

Keywords: Reversed-Phase High Performance Liquid Chromatography; Clindamycin; Metronidazole; Paclitaxel; Tobramycin; Gradient

\section{Introduction}

\section{HPLC}

High Performance Liquid Chromatography (HPLC) is a widely used method to quantify the presence of a pharmaceutical solute in samples. This analytical method involves the injection of a sample into the chromatographic column which then retains the desired sample from the mobile phase. The extracted and bound sample is increased and analyzed through a variety of methods, which involve the detection of a chromophore of the bound agent. HPLC analysis is superior to other forms of UV/Vis quantification due to the process ability to clearly quantitate material as well as remove interference problems inherent in other processes [1]. Despite this distinct advantage, HPLC is a very difficult process with variables such as temperature, column choice, mobile phase composition and mobile phase $\mathrm{pH}$ all being essential to consider [2].

As stated, column choice is a crucial variable to control the efficacy of an HPLC method. Columns are offered in a wide variety of types and sizes, but can generally be divided into normal phase and reverse phase columns. These column names relate to the manner in which the desired solute is removed from the mobile phase and drawn to the analytical column [3]. Reversed phase is by far the more prevalent technology at present and involves hydrophobic columns and aqueous based mobile phases. Commonly used columns in reversed-phase HPLC (RP-HPLC) include C8 and C18 columns. Column choice parameters have been covered in other areas of the literature $[2,3]$.

Following the choice for column type, HPLC methods again fall into two groups based on analytical scheme. These two scheme types are referred to as isocratic and gradient methods. Isocratic methods refer to the use of a single mobile phase composition for the entirety of analysis, whereas gradient methods refer to methods which utilize a changing composition through the process. Isocratic analysis has been considered quicker until recently, however gradient analysis allows higher throughput [4], better resolution [4], and separation of multiple compounds in a single sample [5]. For these reasons gradient analysis is often employed in analytical settings where applicable. When choosing a gradient method, the mobile phase is of great concern. Mobile phases in RP-HPLC usually involve water, acetonitrile, and/or methanol and sometimes a dopant agent such as triethanolamine or triflouroacetic acid is used [2]. Because of the large amounts of variables present in such systems, method development is quite difficult and results in many methods for analyzing the same drug [6]. For this reason, it is important to select and/or develop the best method for a given application's indication.

\section{Discussed antibiotics and published HPLC methods}

The following sections briefly outline the utilized drugs and their relation to HPLC analysis in the lab today. Each of these drugs was chosen for method development based on clinical need.

Cefuroxime: Cefuroxime is a member of the cephalosporin family which displays activity against both Gram-positive and Gram-negative bacteria [7]. It is a well characterized drug with many methods for quantification and has a prominent chromophore with peak UV absorbance in the 270-280 $\mathrm{nm}$ range, making it acceptable for HPLC analysis [8]. The drug is usually used in salt form, with cefuroxime sodium being prevalent. This form is very hydrophilic and for this reason RP-HPLC can be used to quantitate drug presence in solution samples.

Clindamycin: Clindamycin is a lincosamide that is finding a wider use in today's clinic due to the wide range of efficacy for the drug [9]. It can be used to treat infections from staphylococcal to anaerobic

*Corresponding author: Jason Olbrich, Poly-Med, Inc., Technology Center: 51 Technology Drive, Anderson, SC 29625, USA, Tel: 864-328-0008; Fax: (864) 328-0013; E-mail: Jason.Olbrich@Poly-Med.com

Received January 17, 2013; Accepted March 16, 2013; Published March 19 2013

Citation: Olbrich J, Corbett J (2013) Development and Utilization of Reversed Phase High Performance Liquid Chromatography Methods for a Series of Therapeutic Agents. Mod Chem appl 1: 101. doi:10.4172/mca.1000101

Copyright: (c) 2013 Olbrich J. This is an open-access article distributed under the terms of the Creative Commons Attribution License, which permits unrestricted use, distribution, and reproduction in any medium, provided the original author and source are credited. 
to even some protozoal infections [10]. Clindamycin is supplied as a hydrophilic salt, traditionally as a hydrochloride. With this enhanced solubility, RP-HPLC is used with detection around $200 \mathrm{~nm}[9,10]$. Clindamycin methods usually are performed isocratically $[9,10]$, however, gradient methods are useful especially as clindamycin is often used in combination treatments [5].

Dexamethasone: Dexamethasone is a steroid of the glucocorticoid family [1]. These drugs have exhibited effectiveness with treatment of many ocular ailments [11]. Dexamethasone shows the most antiinflammatory activity within this family and is therefore finding many applications within the ocular field [12]. However, excessive levels have been linked with undesirable side effects and so quantification is of the utmost importance [13]. Dexamethasone, like most steroids, is generally lipophilic but can be quantified through HPLC with reverse phase columns. Analysis is commonly performed on C18 columns $[11,12,14]$ and detection is usually between 238 and $255 \mathrm{~nm}[1,11,12]$.

Dicloxacillin: Dicloxacillin is a modern member of the penicillin family. It is penicillinase resistant allowing for use in indications where other members of the group are not effective [15]. It is a highly polar compound which causes difficulty with extraction in many processes [16]. Previous work with this drug has involved reversed phase columns and utilized detection wavelengths between 220 and $240 \mathrm{~nm}$ [15-18].

Doxycycline: This common tetracycline drug is used widely due to its broad spectrum of activity [19]. It has been well defined pharmacokinetically with a reliable absorption and long half-life [20]. Recently, doxycycline has exhibited inhibition of enzymatic activity, such as that of the matrix metalloproteinase's (MMP's) [21]. For these reasons pared with wide patient tolerance, this drug has been used in many indications and HPLC methods have been developed for quantification [19-21]. These methods involve a wide range of detection wavelengths with $350 \mathrm{~nm}$ being the most common [19].

Metronidazole: This cytostatic drug has a wide range of uses within the clinic. Metronidazole is prescribed for conditions ranging from rosacea to bacterial vaginosis [22]. The drug has also been shown to sensitize tumors to radiotherapy, opening its use in oncology [23]. For these conditions, the drug is often carried within a gel [24], with release from the gel being an important experimental variable. For this reason, HPLC is often employed as the drug salt is water soluble, has a strong chromophore, and is used in the described wide variety of applications. Detection for this drug is performed over a wide range from 250 to 350 nm [22-24].

Oxymetazoline: Oxymetazoline is a widely used non-prescription drug which acts as a vasoconstrictor [25]. It is generally used in the nasal cavity as a decongestant $[25,26]$ and can be used in combination therapies for a wide variety of ear, nose and throat disorders [25-27]. The drug has a relatively low molecular weight, much of which is contained within the chromophore structure itself. This drug is often used in ointments and sprays and therefore ability to quantify release through HPLC is of great value. Analysis is traditionally performed using a C18 column with detection at $230 \mathrm{~nm}$ [25-27].

Paclitaxel: Taxanes and paclitaxel in particular have taken a leading role in chemotherapy over the recent decade. This expansion of use is largely due to the efficacy of paclitaxel in treatment of a wide range of solid tumors [28]. This drug moves to block mitotic activity through interference with the formation of microtubules [29], thus preventing cancer cell division. However, due to the prevalence of significant side effects, paclitaxel is almost always used in a comparatively low dose to other bioactive agents [30]. This need for a precise window of treatment combined with the important nature of the drugs indications make paclitaxel an important drug for quantification. HPLC is uniquely suited for the low levels of drug used as well as the common use of paclitaxel with other drugs and excipients such as Chremophor EL $^{\circledR}$ [28-31]. The hydrophobic nature of the drug does necessitate different mobile phase ratios than other drugs described, but hydrophobic columns can be used and detection is usually around $230 \mathrm{~nm}$ [28-31].

Vancomycin: Vancomycin is a glycopeptide drug which has come to be used for critical infections where drugs of lesser efficacy cannot be utilized or in instances of drug resistance [32]. Specifically, vancomycin is used clinically to treat methicillin resistant Staphylococcus aureus (MRSA) [33]. Vancomycin is a relatively large molecular weight drug with many chromophores and is also hydrophilic [34]. These characteristics lend themselves to RP-HPLC with many methods described in the literature with detection traditionally in the 220-240 nm range [32-34].

Tobramycin: Many serious infections within the body require specialized antibiotics. Tobramycin is often used to combat difficult infection such as osteomyelitis [35] and other serious gram negative bacillary infections [36]. This aminoglycoside chemically is similar to a sugar complex but presents challenges for HPLC analysis as it lacks a chromophore [35]. Because of this, pre-column derivatization techniques are often employed to make this drug visible to an associated UV detector [36,37]. The method utilized in this paper is similar to presented by Lai of Varian Chromatography Systems [37].

\section{Materials and Methods}

\section{Materials}

Pharmaceutical drugs and triflouroacetic acid (TFA) were acquired from Sigma-Aldrich (St. Louis, MO). Acetonitrile (ACN) was purchased from Fisher Chemical (Waltham MA). HPLC grade water was prepared in lab using a Barnstead E-pure system series 582.

\section{HPLC system}

A single HPLC instrument was utilized for the detection of all drugs, allowing for limited maintenance and ease of work flow interruption between projects. Column utilized was a Waters Symmetry ${ }^{\circledR}$ C18 5 um $4.6 \times 150 \mathrm{~mm}$ column. Pump was a Waters 1525 binary HPLC pump. Pump and column were coupled with a Water 2996 Photodiode Array Detector (PDA). All mobile phases were first filtered through a Waters four channel in-line degasser prior to entrance into pump housing. Mobile Phases utilized were HPLC grade water with $0.1 \%$ TFA and ACN with $0.1 \%$ TFA. A Waters 717 plus autosampler was employed to allow multiple sample injection. Software for analysis was Waters Empower Pro. Flow rate was set at $1 \mathrm{ml} / \mathrm{min}$. Methods herein are described by percentage of ACN mobile phase, omitting the complimentary percentage of HPLC composition in the mobile phase.

\section{Method development: A general outline}

Methods for each drug were generated specifically for the provided HPLC apparatus. A drug solution of greater than $0.1 \mathrm{mg} / \mathrm{ml}$ was prepared in mobile phase solution depending on the solubility of the given drug. Sample was then analyzed on HPLC apparatus through a gradient method of $5 \% \mathrm{ACN}$ to $95 \% \mathrm{ACN}$ over a period of 20 minutes. The $5 \%$ to $95 \%$ range was chosen so as to limit usage of the end ranges as this will enhance the lifetime of the column. This HPLC trace provides a general range for the determination of the second method attempt. Based on the elution time the original run the HPLC peak presents, a second run is prepared with a range of $15 \%$ change in ACN over a 
Citation: Olbrich J, Corbett J (2013) Development and Utilization of Reversed Phase High Performance Liquid Chromatography Methods for a Series of Therapeutic Agents. Mod Chem appl 1: 101. doi:10.4172/mca.1000101

space of 10 minutes. Based on presentation of peak within this second method, mobile phase composition was adjusted until the major eluent peak presented more than 5 minutes after the solvent injection peak, to be assured of peak resolution from the solvent front. Analytical wavelength for each drug was determined as the wavelength which corresponded with the maximum absorbance response compared to baseline noise for the sample peak as provided by the attached PDA detector. Following development of method, process was analyzed for reproducibility through generation of a standard curve consisting of at least five varying injection quantities and the confirmation of consistency through a $\mathrm{R}^{2}$ value of greater than 0.99 when comparing micrograms injected and absorbance reported.

\section{Results and Discussion}

Results are presented for each drug subsequently. For each drug, methodology, analytical wavelength and standard curve are provided.

\section{Cefuroxime}

Cefuroxime sodium salt was determined to be best analyzed through the gradient method of 20 to $35 \%$ ACN over a time of 10 minutes. With this method, a peak was found at approximately 7.25 minutes. This peak was also determined to have a best absorbance for analysis at $275 \mathrm{~nm}$. A standard curve was then prepared of a sample solution injected at different injection volumes, resulting in an $\mathrm{R}^{2}$ value of 0.9999. Prepared standard curve is seen in figure 1.

\section{Clindamycin}

Clindamycin hydrochloride analysis method was developed to be $20 \%$ to $35 \%$ ACN over a period of 10 minutes. With this method, a peak was found at approximately 8.15 minutes. This peak was also determined to have a best absorbance for analysis at $210 \mathrm{~nm}$. A standard curve was then prepared of a sample solution injected at different injection volumes, resulting in an $\mathrm{R}^{2}$ value of 0.9999 . Prepared standard curve is seen in figure 2 .

\section{Dexamethasone}

Dexamethasone sodium phosphate was determined to be best analyzed through the gradient method of 25 to $40 \%$ ACN over a time of 10 minutes. With this method, a peak was found at approximately 8.15 minutes. This peak was also determined to have a best absorbance for analysis at $240 \mathrm{~nm}$. A standard curve was then prepared of a sample solution injected at different injection volumes, resulting in an $\mathrm{R}^{2}$ value of 0.999 . Prepared standard curve is seen in figure 3.

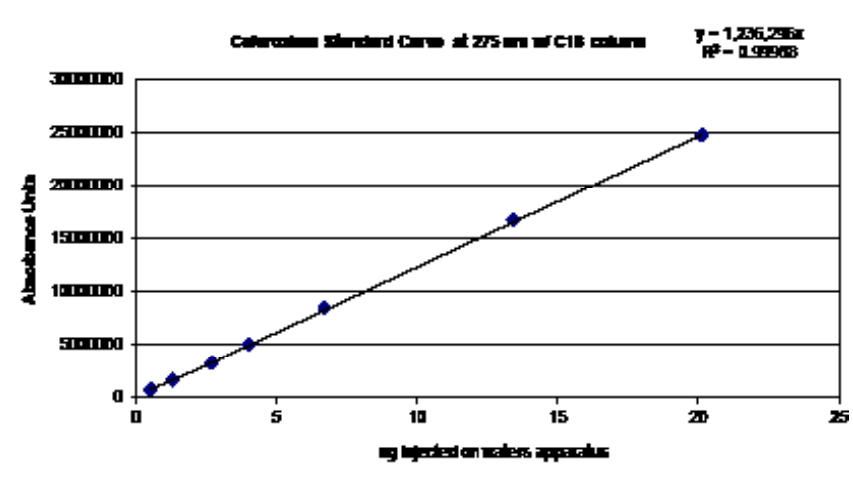

Figure 1: Cefuroxime standard curve.

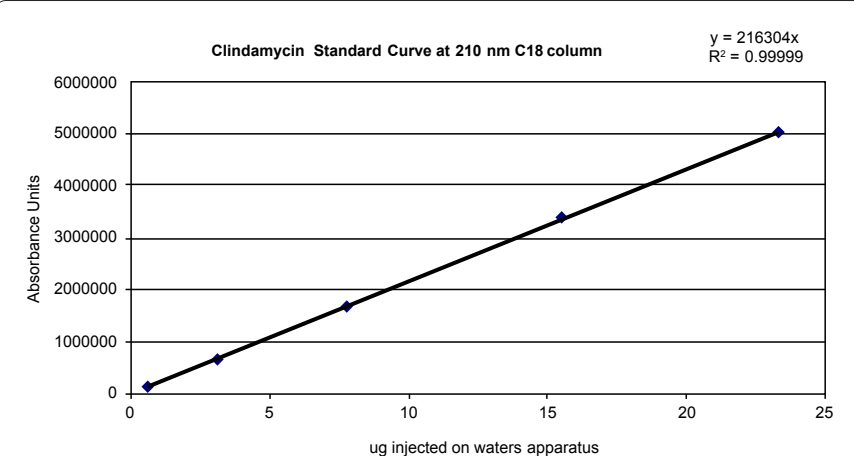

Figure 2: Clindamycin standard curve.

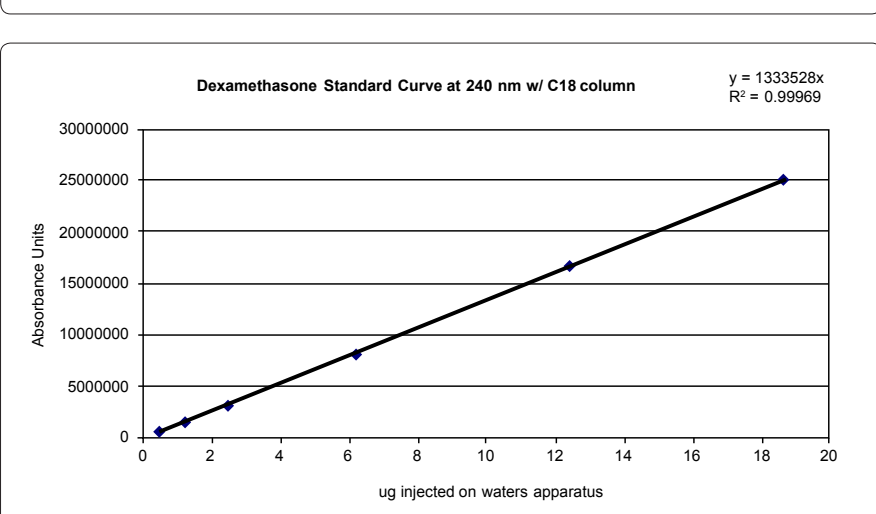

Figure 3: Dexamethasone standard curve.

\section{Dicloxacillin}

Dicloxacillin salt monohydrate was determined to be best analyzed through the gradient method of 45 to $60 \%$ ACN over a time of 10 minutes. With this method, a peak was found at approximately 7.33 minutes. This peak was also determined to have a best absorbance for analysis at $225 \mathrm{~nm}$. A standard curve was then prepared of a sample solution injected at different injection volumes, resulting in an $\mathrm{R}^{2}$ value of 0.999 . Prepared standard curve is seen in figure 4 .

\section{Doxycycline}

Doxycycline hyclate was determined to be best analyzed through the gradient method of 23 to $38 \% \mathrm{ACN}$ over a time of 10 minutes. With this method, a peak was found at approximately 7.85 minutes. This peak was also determined to have a best absorbance for analysis at $350 \mathrm{~nm}$. A standard curve was then prepared of a sample solution injected at different injection volumes, resulting in an $\mathrm{R}^{2}$ value of 0.999 . Prepared standard curve is seen in figure 5 .

\section{Metronidazole}

Metronidazole free base was determined to be best analyzed through an isocratic method of $3 \%$ ACN over a time of 10 minutes. Gradient methods proved difficult due to the high water solubility of the drug, and a percentage outside of the 5 to $95 \%$ preferred range was required. This method exhibits the effectiveness of isocratic methods with the described HPLC apparatus. With this method, a peak was found at approximately 7.30 minutes. This peak was also determined to have a best absorbance for analysis at $275 \mathrm{~nm}$. A standard curve was then prepared of a sample solution injected at different injection volumes, resulting in an $\mathrm{R}^{2}$ value of 0.999 . Prepared standard curve is seen in figure 6. 
Citation: Olbrich J, Corbett J (2013) Development and Utilization of Reversed Phase High Performance Liquid Chromatography Methods for a Series of Therapeutic Agents. Mod Chem appl 1: 101. doi:10.4172/mca.1000101

Page 4 of 6

\section{Oxymetazoline}

Oxymetazoline hydrochloride was determined to be best analyzed through the gradient method of 25 to $40 \%$ ACN over a time of 10 minutes. With this method, a peak was found at approximately 8.10 minutes. This peak was also determined to have a best absorbance for analysis at $210 \mathrm{~nm}$. A standard curve was then prepared of a sample solution injected at different injection volumes, resulting in an $\mathrm{R}^{2}$ value of 0.999. Prepared standard curve is seen in figure 7.

\section{Paclitaxel}

Paclitaxel free base, free of excipients, was determined to be best analyzed through the gradient method of 50 to $65 \%$ ACN over a time of 10 minutes. With this method, a peak was found at approximately 6.55 minutes. This peak was also determined to have a best absorbance for analysis at $230 \mathrm{~nm}$. A standard curve was then prepared of a sample solution injected at different injection volumes, resulting in an $\mathrm{R}^{2}$ value of 0.999 . Prepared standard curve is seen in figure 8 .

\section{Vancomycin}

Vancomycin hydrochloride was determined to be best analyzed through the gradient method of 10 to $25 \%$ ACN over a time of 10 minutes. With this method, a peak was found at approximately 6.78 minutes. This peak was also determined to have a best absorbance for analysis at $210 \mathrm{~nm}$. A standard curve was then prepared of a sample solution injected at different injection volumes, resulting in an $\mathrm{R}^{2}$ value of 0.999. Prepared standard curve is seen in figure 9 .

\section{Tobramycin}

Tobramycin sulfate salt was successfully prepared for HPLC

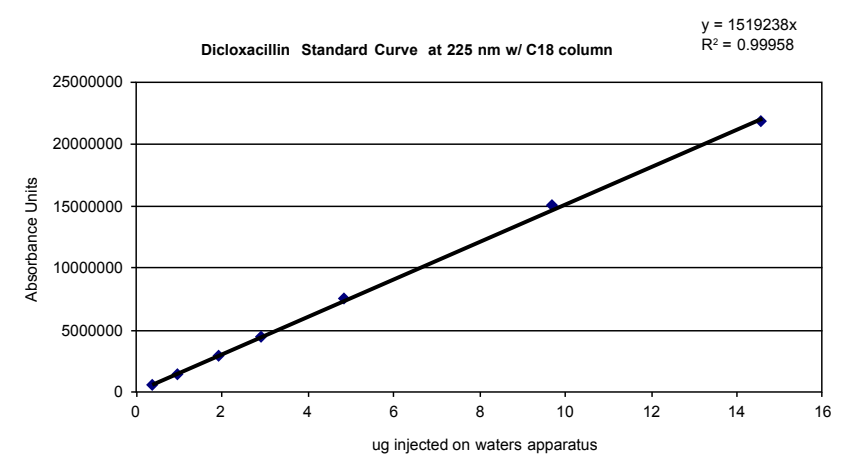

Figure 4: Dicloxacillin standard curve.

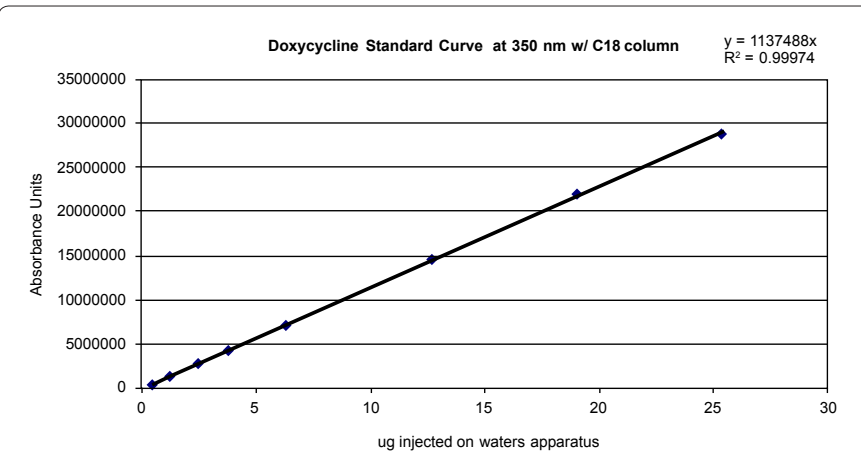

Figure 5: Doxycycline standard curve.

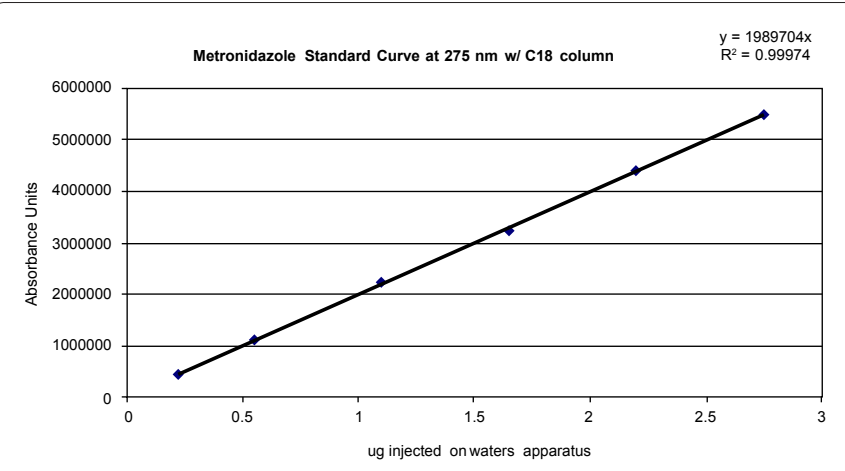

Figure 6: Metronidazole standard curve.

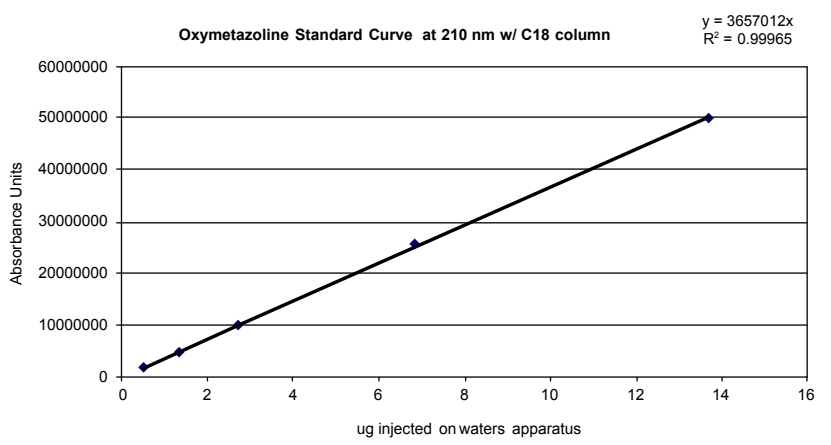

Figure 7: Oxymetazoline standard curve.

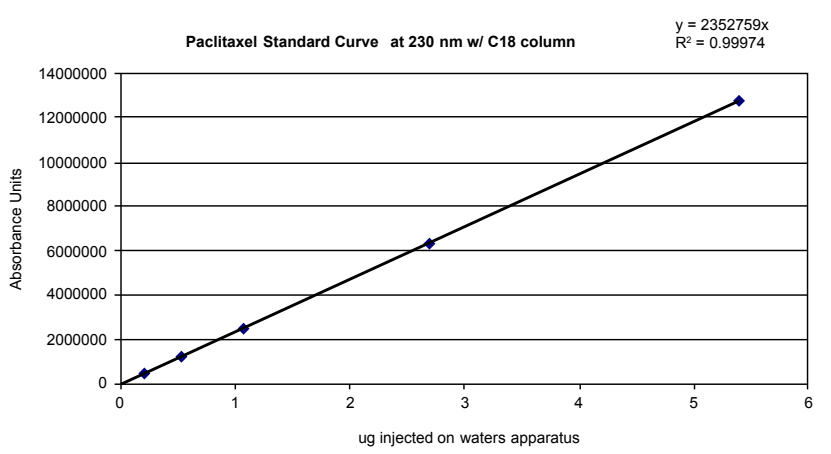

Figure 8: Paclitaxel standard curve.

analysis through a pre-column derivatization as described [37]. A precolumn derivatization was required due to the lack of a chromophore on the tobramycin structure. Analysis of the tobramycin complex was determined to be best performed through the gradient method of 5 to 95\% ACN over a time of 20 minutes. With this method, a peak was found at approximately 6.15 minutes. This peak was also determined to have a best absorbance for analysis at $254 \mathrm{~nm}$. Other peaks present in chromatogram were omitted from standard curve analysis. A standard curve was prepared of a sample solution injected at different injection volumes, resulting in an $\mathrm{R}^{2}$ value of 0.99 . Prepared standard curve is seen in figure 10 .

As the reaction utilized to prepare the HPLC visible complex is time sensitive, analysis of tobramycin visibility degradation was performed. 
Citation: Olbrich J, Corbett J (2013) Development and Utilization of Reversed Phase High Performance Liquid Chromatography Methods for a Series of Therapeutic Agents. Mod Chem appl 1: 101. doi:10.4172/mca.1000101

Page 5 of 6

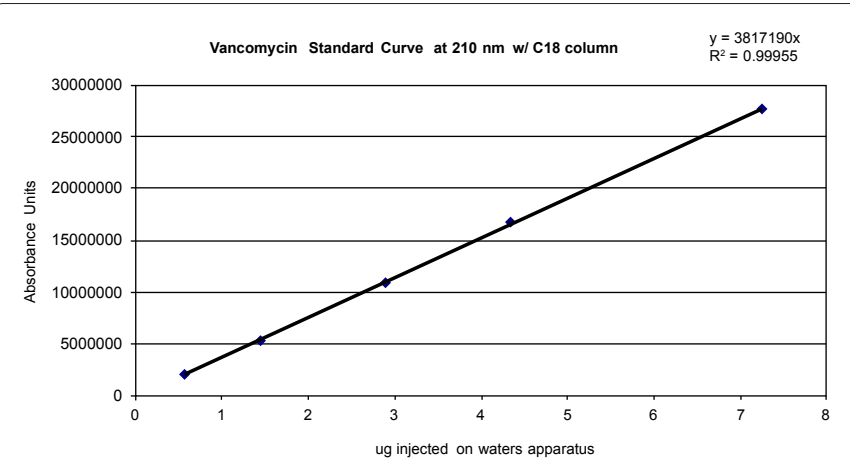

Figure 9: Vancomycin standard curve.

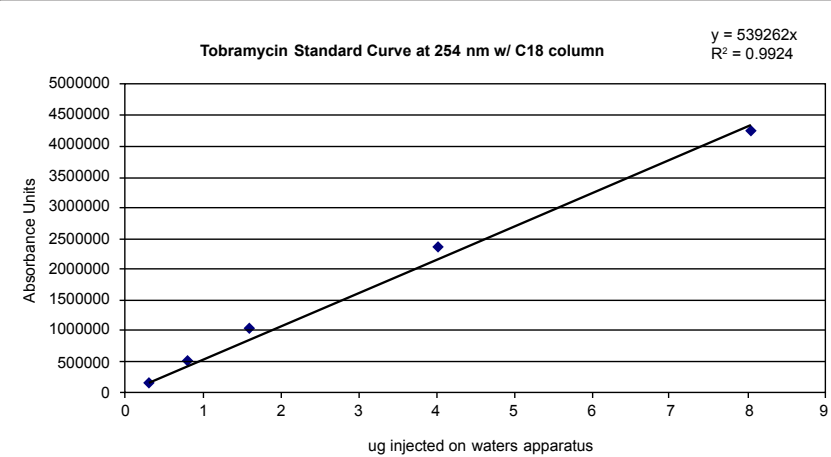

Figure 10: Tobramycin standard curve.

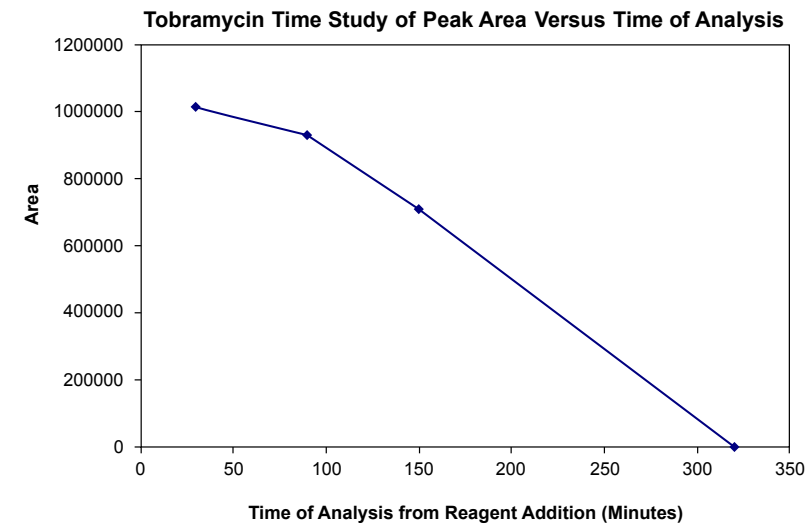

Figure 11: Tobramycin time complex stability study.

A sample was tested at 30, 90, 150 and 320 minutes for response. Results can be seen in figure 11. From this information it can be determined that analysis should take place in the first hour following the pre-column derivatization to achieve the best results and a repeatable method.

\section{Conclusion}

As can be seen from the provided data, multiple drugs can be analyzed from a single HPLC instrument not apparatus. Also, each drug has a well-defined and reproducible analytical method presented. These methods have been determined to be efficient for the drug at hand, and where applicable gradient methods allow for separation and detection of multiple compounds if called for by the experiment.
Drugs which are both hydrophobic and hydrophilic in nature have been quantified using a reverse-phase system. A method of detection for the aminoglycoside tobramycin is advanced and verified for the system. With a well-planned and executed methodology, HPLC can be a powerful tool for the detection of a wide range of antibiotics while requiring only a single apparatus.

\section{References}

1. Garcia CV, Breier AR, Steppe M, Schapoval EE, Oppe TP (2003) Determination of dexamethasone acetate in cream by HPLC. J Pharm Biomed Anal 31: 597 600

2. Medenica M, Ivanovic D, Markovic S, Malenovic A, Misljenovic D (2003) Optimization of an RP-HPLC Method for Drug Control Analysis. Journal of Liquid Chromatography \& Related Technologies 26: 3401-3412.

3. Ahuja S (2008) A Strategy for Developing HPLC Methods for Chiral Drugs. The Application Notebook. 70-79.

4. Joseph MJ (2003) Fast HPLC Analysis of Antibiotics Using Columns with Sub Two-Micron Particles. LC-GC North America, The Application Notebook 21: 41

5. Thomas LA, Bizikova T, Minihan AC (2011) In vitro elution and antibacterial activity of clindamycin, amikacin and vancomycin from R-gel polymer. Vet Surg 40: 774-780.

6. Dejaegher B, Jimidar M, De Smet M, Cockaerts P, Smeyers-Verbeke J, et, al (2006) Improving method capability of a drug substance HPLC assay. J Pharm Biomed Anal 42: 155-170.

7. Piva G, Farin D, Gozlan I, Kitzes-Cohen R (2000) HPLC method for determination of cefuroxime in plasma. Chromatographia 51: 154-156.

8. Vieira DC, Salgado HR (2011) Comparison of HPLC and UV spectrophotometric methods for the determination of cefuroxime sodium in pharmaceutical products. J Chromatogr Sci 49: 508-511.

9. Batzias GC, Delis GA, Athanasiou LV (2005) Clindamycin bioavailability and pharmacokinetics following oral administration of clindamycin hydrochloride capsules in dogs. Vet J 170: 339-345.

10. Batzias GC, Delis GA, Koutsoviti-Papadopoulou M (2004) A new HPLC/UV method for the determination of clindamycin in dog blood serum. J Pharm Biomed Anal 35: 545-554.

11. Gomez-Gaete C, Tsapis N, Besnard M, Bochot A, Fattal E (2006) Encapsulation of dexamethasone into biodegradable polymeric nanoparticles. Int J Pharm 331: 153-159.

12. Kumar V, Mostafa S, Kayo MW, Goldberg EP, Derendorf H (2006) HPLC Determination of dexamethasone in human plasma and its application to an in vitro release study from endovascular stents. Pharmazie 61: 908-911.

13. Song Y, Park J, Kim J, Kim C (2004) HPLC Determination of Dexamethasone in Human Plasma. Journal of Liquid Chromatography \& Related technologies 27: 2293-2306.

14. Moya-Ortega $M$, Messner $M$, Jansook $P$, Nielsen T, Wintgens $V$, et al. (2011) Drug loading in cyclodextrin polymers: dexamethasone model drug. Journal of Inclusion Phenomena and Macrocyclic Chemistry 69: 377-382.

15. Dhoka MV, Sandage SJ, Dumbre SC (2010) Simultaneous determination of cefixime trihydrate and dicloxacillin sodium in pharmaceutical dosage form by reversed-phase high-performance liquid chromatography. J AOAC Int 93: 531535

16. Alderete O, Gonzalez-Esquivel DF, Del Rivero LM, Castro Torres N (2004) Liquid chromatographic assay for dicloxacillin in plasma. J Chromatogr B Analyt Biomed Life Sci 805: 353-356.

17. Samanidou VF, Nisyriou SA, Papadoyannis IN (2007) Development and validation of an HPLC method for the determination of penicillin antibiotics residues in bovine muscle according to the European Union decision 2002/657/ EC. J Sep Sci 30: 3193-3201.

18. Samanidou VF, Giannakis DE, Papadaki A (2009) Development and validation of an HPLC method for the determination of seven penicillin antibiotics in veterinary drugs and bovine blood plasma. J Sep Sci 32: 1302-1311.

19. Kogawa AC, Salgado HR (2012) Quantification of Doxycycline Hyclate in Tablets by HPLC-UV Method. J Chromatog Sci.

20. Mitic S, Miletic G, Kostic D, Dokic D, Arisc B et, al. (2008) A rapid and 
Citation: Olbrich J, Corbett J (2013) Development and Utilization of Reversed Phase High Performance Liquid Chromatography Methods for a Series of Therapeutic Agents. Mod Chem appl 1: 101. doi:10.4172/mca.1000101

reliable determination of doxycycline hyclate by HPLC with UV detection in pharmaceutical samples. Journal of the Serbian Chemical Society 73: 665-671.

21. Skulason S, Ingolfsson E, Kristmundsdottir T (2003) Development of a simple HPLC method for separation of doxycycline and its degradation products. $J$ Pharm Biomed Anal 33: 667-672.

22. Tashtoush BM, Jacobson EL, Jacobson MK (2008) Validation of a simple and rapid HPLC method for determination of metronidazole in dermatological formulations. Drug Dev Ind Pharm 34: 840-844.

23. Akay C, Ozkan SA, Senturk Z, Cevheroglu S (2002) Simultaneous determination of metronidazole and miconazole in pharmaceutical dosage forms by RPHPLC. Farmaco 57: 953-957.

24. Miani PK, do Nascimento C, Sato S, Filho AV, da Fonseca MJ, et al. (2012) In vivo evaluation of a metronidazole-containing gel for the adjuvant treatment of chronic periodontitis: preliminary results. Eur J Clin Microbiol Infect Dis 31 : 1611-1618.

25. Mahajan MK, Uttamsingh V, Gan LS, Leduc B, Williams DA (2011) Identification and Characterization of oxymetazoline glucuonidation in human liver microsomes: evidence of the involvement of UGT1A9. J Pharm Sci 100: 784793.

26. Sudsakorn S, Kaplan L, Williams DA (2006) Simultaneous determination of triamcinolone acetonide and oxymetazoline hydrochloride in nasal spray formulations by HPLC. J Pharm Biomed Anal 40: 1273-1280.

27. Golubitskii GB, Basova EM, Ivanov VM (2008) Application of gradient highperformance liquid chromatography to the analysis of some multicomponent pharmaceutical preparations. J Anal Chem 63: 875-880.

28. Badea I, Ciutaru D, Lazar L, Nicolescu D, Tudose A (2004) Rapid HPLC Method for the determination of paclitaxel in pharmaceutical forms without separation. 34: 501-507.
29. Kim SC, Yu J, Lee JW, Park ES, Chi SC (2005) Sensitive HPLC Method for quantitation of paclitaxel (Genexol in biological samples with application to preclinical pharmacokinetics and biodistribution. J Pharm Biomed Anal 39: 170-176.

30. Yonemoto H, Ogino S, Nakashima MN, Wada M, Nakashima K (2007) Determination of paclitaxel in human and rat blood samples after administration of low dose paclitaxel by HPLC-UV detection. Biomed Chromatogr 21: 310317.

31. Torne SJ, Ansari KA, Vavia PR, Trotta F, Cavalli R (2010) Enhanced ora paclitaxel bioavailability after administration of paclitaxel-loaded nanosponges. Drug Deliv 17: 419-425.

32. Jesus Valle MJ, Lopez FG, Navarro AS (2008) Development and validation of an HPLC method for vancomycin and its application to a pharmacokinetic study. J Pharm Biomed Anal 48: 835-839.

33. Liu M, Hu C (2006) Simultaneous Determination of the Purity and Potency of Vancomycin and Norvancomycin by HPLC. Chromatographia 65: 203-207.

34. Frick P, Nagy Z, Fekete J, Kettrup A, Gebefugi I (2001) Reverse Phase HPLC Method for Determination of Vancomycin in Influenza Vaccine. J Liq Chromatogr Relat Technol 24: 497-507.

35. Valentini F, Buldini PL, Landi E, Tampieri A, Tonelli D (2008) HPLC determination of tobramycin in a simulated body fluid. Microchemical Journal 90: 113-117.

36. He S, Chen Q, Sun Y, Zhu Y, Luo L, et, al. (2011) Determination of tobramycin in soil by HPLC with ultrasonic-assisted extraction and solid phase-extraction. J Chromatogr B Analyt Tecnol Biomed Life Sci 879: 901-907.

37. Lai F, Sheehan T (1992) Enhancement of detection sensitivity and cleanup selectivity for tobramycin through pre-column derivatization. J Chromatogr 609 : 173-179. 